\title{
Elastic properties of muscles measured at the elbow in man: I. normal controls
}

\author{
ALLEN W WIEGNER, RAY L WATTS* \\ From the Clinical Neurophysiology Laboratory and Neurology Service, Massachusetts General Hospital and \\ Harvard Medical School, Boston, USA
}

SUMMARY Passive elastic stiffness of muscle acting at the elbow was assessed in 19 normal subjects by measuring displacements produced by a torque motor acting at the joint. Stiffness ranged from 0.40 to $1.8 \mathrm{Nm} /$ radian and was strongly correlated $(\mathrm{r}=0.85)$ with upper arm volume, allowing us to define a "normal" range for stiffness when corrected for arm volume. In addition, the angle of the elbow with the arm fully relaxed and no external torque applied ("neutral" angle) was found to be $107^{\circ} \pm 10^{\circ}$. Thus, we have quantified resting stiffness or "tone" in the arm and provided normal data for comparison with patients with pathophysiological conditions such as rigidity or spasticity.

Although one usually considers muscle activity as underlying movement, recent studies of the control of limb movements have recognised the importance of passive elastic and viscous properties of muscles for their effects on limb positioning, ${ }^{1-3}$ postural stability, ${ }^{4}$ ballistic movements, ${ }^{5}$ and in the evaluation of patients with pathologically increased muscle "tone". 6 Lakie and colleagues ${ }^{4}$ found an increased stiffness at resting, as compared to moving, joints of humans which they and Wiegner ${ }^{7}$ have shown to be due at least in part to the short range stiffness properties of passive muscle. Electromyograms (EMG) recorded from the antagonist muscle during ballistic movements of a given size vary as a function of the ending position of those movements, reflecting a varying contribution of passive muscle properties to limb deceleration. ${ }^{5}$

Clinical "tone" as assessed during physical examination is used as a diagnostic sign and as a means of following the progress of treatment of disorders which involve spasticity or rigidity. A clinician's evaluation of enhanced tone on a scale of mild to severe may be consistent in a given patient, but it is not an ideal system for following changes over time, or when making quantitative comparisons between examiners. Thus a number of attempts to quantify tone have

*Present address: Laboratory of Neurophysiology, NIMH, Bethesda, MD 20205, USA.

Address for reprint requests: Dr Allen W Wiegner, Clinical Neurophysiology Laboratory, Massachusetts General Hospital, Boston, Mass, 02114, USA.

Received 2 July 1985 and in revised form 9 December 1985. Accepted 15 December 1985 been described in the literature (see Watts et $a l^{8}$ ), in each case with the aim of evaluating a particular class of patients. With spasticity or with Parkinsonian patients who have assumed a more flexed posture, it is not obvious whether increased tone is due to heightened reflexes alone or whether inactivity-related muscle contractures also play a role. ${ }^{9}$ Such contractures can affect the working length of a muscle, altering the resting limb position and passive torque-angle relation at the joint(s) at which the muscle acts. Therefore we have measured the resting elbow angle and the compliance at the elbow in a series of normal subjects, with the dual purposes of finding a convenient, quantitative measure of compliance as well as defining "normal" compliance and elbow angle for later comparison with patient groups having heightened tone or rigidity.

\section{Methods}

Subjects were seated in a chair with the right upper arm supported comfortably on a table at shoulder height. The forearm, wrist, and semi-pronated hand were strapped to the lever arm of a printed motor (Axem MC19S) mounted below the table. Each subject was positioned so that the axis of rotation of the elbow joint was co-axial with the motor shaft. Lever arm position was measured with a magnetoresistive potentiometer (Midori CP-2U-33) on the motor shaft. Motor torque was set under computer control; torque in the lever arm was measured with strain gauges.mounted on the lever arm. Friction torque in the motor was specified as $0.1 \mathrm{Nm}$, and this value was observed in practice; the damping constant of the 

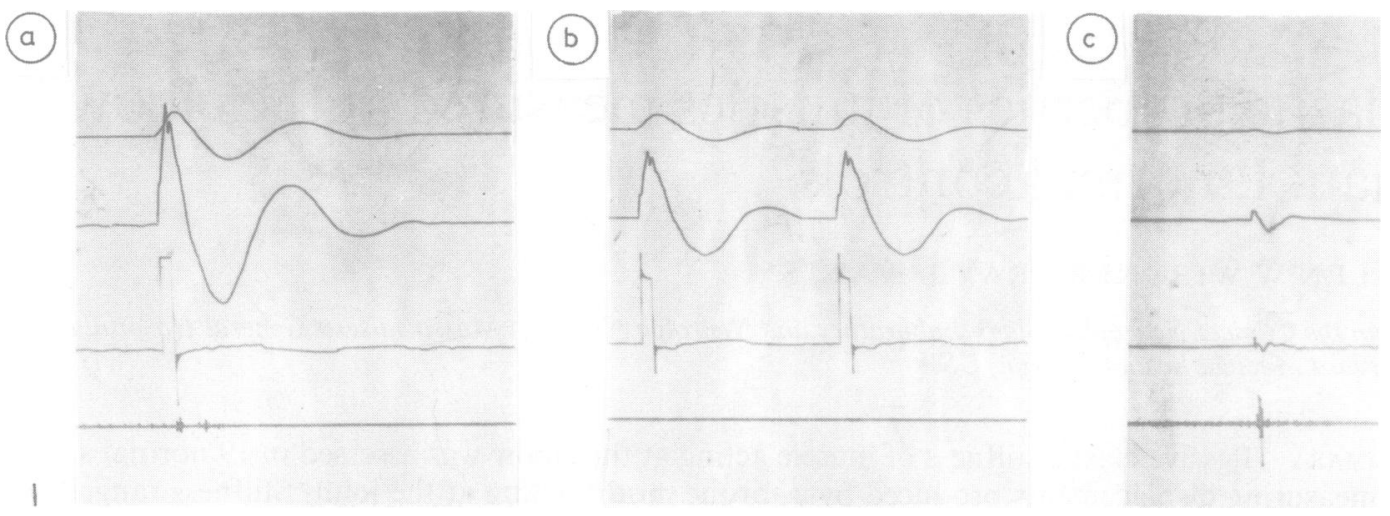

Fig 1 Responses of the relaxed arm to imposed torque pulses. Traces are, from top, elbow angle (cal $=35$ ); angular velocity. $(c a l=2 \mathrm{rad} / \mathrm{s}) ;$ torque $(\mathrm{c}$ al $=5 \mathrm{Nm})$; and brachioradialis $E M G$ measured with Copeland-Davies electrodes $(\mathrm{c} a \mathrm{l}=250 \mu \mathrm{V})$. time cal $=0.5 \mathrm{~s}$. Note stretch reflex EMG response with torque pulse of $7 \mathrm{Nm}$ (left) which is absent at $4 \mathrm{Nm}$ (centre). A stretch reflex can also be elicited by tapping the hiceps tendon (right).

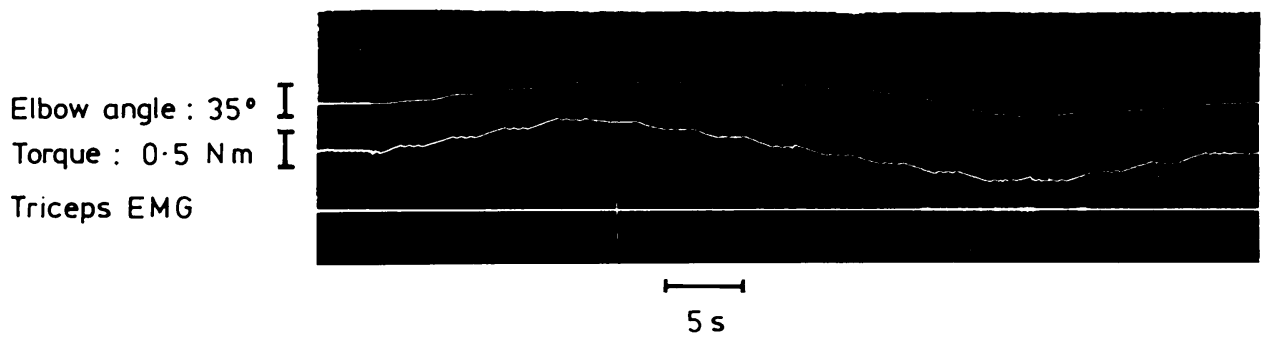

Fig 2 A single trial showing extension and flexion at the elhow. Small ripples seen in the torque trace we're part of the input pattern to the torque motor. The EMG record is silent except for a random noise spike.

motor was specified at $0.0008 \mathrm{Nm} \mathrm{s} / \mathrm{rad}$ and was ignored.

A series of preliminary experiments was performed to examine the relationship between limb velocity and reflex contraction of muscles in the relaxed limb. Torque pulses were applied to the relaxed arm while in its neutral position (the position to which it would return when manually deflected by the experimenter and allowed to move freely until it stopped). Torque pulses ranged from 1 to $7 \mathrm{Nm}$ in amplitude and were of $100 \mathrm{~ms}$ duration, with rise and fall times of $10 \mathrm{~ms}$. Resulting trajectories of the limb were repeatable and consisted of a linear increase in angular velocity during the duration of the pulse, as would be expected of a mass subjected to constant torque, followed by a damped oscillatory displacement pattern (fig 1). Maximum velocity was proportional to torque pulse amplitude in a given subject. To assess muscle activity we monitored the EMG of each of the muscles acting at the elbow (three heads of triceps, two heads of biceps, brachialis, brachioradialis, anconeus) in at least one of three subjects. EMG was recorded using surface, Copeland-Davies, or intramuscular wire electrodes, the latter used on the deep brachialis. Arm position, velocity, and EMG were recorded on a TECA Instagraph which records on light-sensitive paper (Kodak type 1895). The magnitude of reflex responses, as measured by EMG, was a function of the magnitude of the applied torque (and hence the resulting velocity of the limb) and of the subject's state of relaxation. As a subject learned to relax more completely, reflex responses became less frequent. Occasionally a startle response produced a large reflex contraction. Reflex responses were not seen when peak velocities at the elbow joint were less than $100^{\circ} / \mathrm{s}$; this corresponded to a torque pulse amplitude of approximately $4 \mathrm{Nm}$.

In the formal series of experiments, a computer (Digital Equipment Corporation 11/23) provided a torque pattern to the motor so that the elbow was first extended and then flexed during a single trial lasting one minute (fig 2). This torque pattern consisted of a series of four positive (extending) levels of torque (levels 1-4) and four negative (flexing) levels of torque 
(levels 5-8), with the difference between levels selected by the experimenter so that the total range of movement was approximately $60^{\circ}$. Small oscillations in torque were superimposed at each torque level to minimise effects of "sticktion" in the apparatus and thixotropic effects in the passive limb. ${ }^{4}$ To insure that reflex activity would not confound the results of our regular experiments, the rate of change of torque was selected to be less than $0.11 \mathrm{Nm} / \mathrm{s}$, which produced velocities of less than $20^{\circ} / \mathrm{s}$. This gave us a safety factor of five below the minimum velocity values at which reflex responses were seen in the preliminary experiments.

The friction torque in the apparatus $(0 \cdot 1 \mathrm{Nm})$, while small, was not negligible compared to applied torques. Therefore, experiments were repeated both in the presence of friction torque and when friction torque was cancelled electronically within the torque servosystem. A fixed extra current could be supplied to the motor, based on the direction of motion, of a magnitude so that the resulting extra motor torque would just cancel friction torque.

Subjects were instructed to relax, close their eyes, and neither help nor hinder the movement of their arm. The torque servosystem was set for either regular or frictionless operation, and the block of trials which was performed first alternated from subject to subject. Triceps EMG was monitored with surface electrodes during all experiments and audible feedback provided to the subject to facilitate relaxation prior to and throughout the experiment. In general, once a subject succeeded in totally relaxing his arm, no EMG was elicited during the study. However, certain subjects demonstrated a persistent shortening reaction $^{10}$ in the triceps during elbow extension. That is, the muscle being shortened underwent a period of contraction, which often persisted as long as the muscle remained in its shortened state. Subjects who showed such shortening reactions, made voluntary movements, or fell asleep, were removed from the study.

Torque and position were sampled and stored in the computer at a rate of $50 \mathrm{~Hz}$. Only data acquired during the latter part of the dwell at each torque level were used, in order to allow position to stabilise after each change in torque. Position data at each level (baseline, 1-3, 5-7) obtained during flexion and extension were averaged to take into account the effects of limb hysteresis (fig 3). Data at levels 4 and 8 , the maximum extension and flexion excursions of the limb, were ignored. Ten trials were recorded from each subject; position and measured torque were averaged at each level. A linear regression was then performed on the points obtained at the remaining levels in order to obtain a measure of limb compliance in degrees $/ \mathrm{Nm}$. Stiffness was calculated by inverting

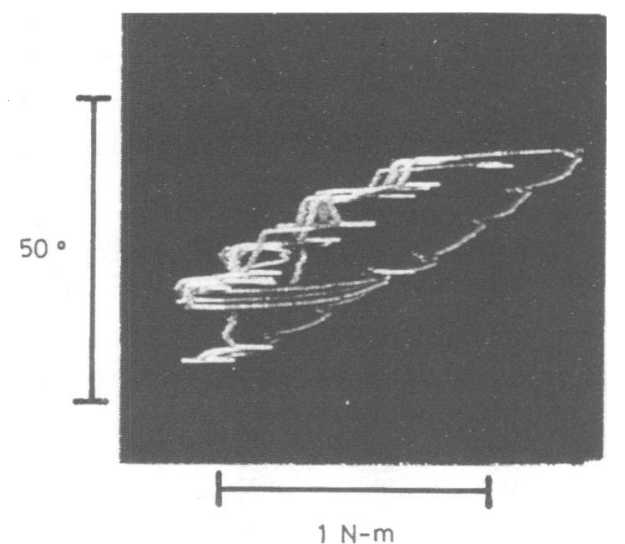

Fig 3 A plot of elbow angle versus measured torque during a block of ten trials. The area within the circles, traversed in a counterclockwise manner, represents work done in moving the resting limb and apparatus.

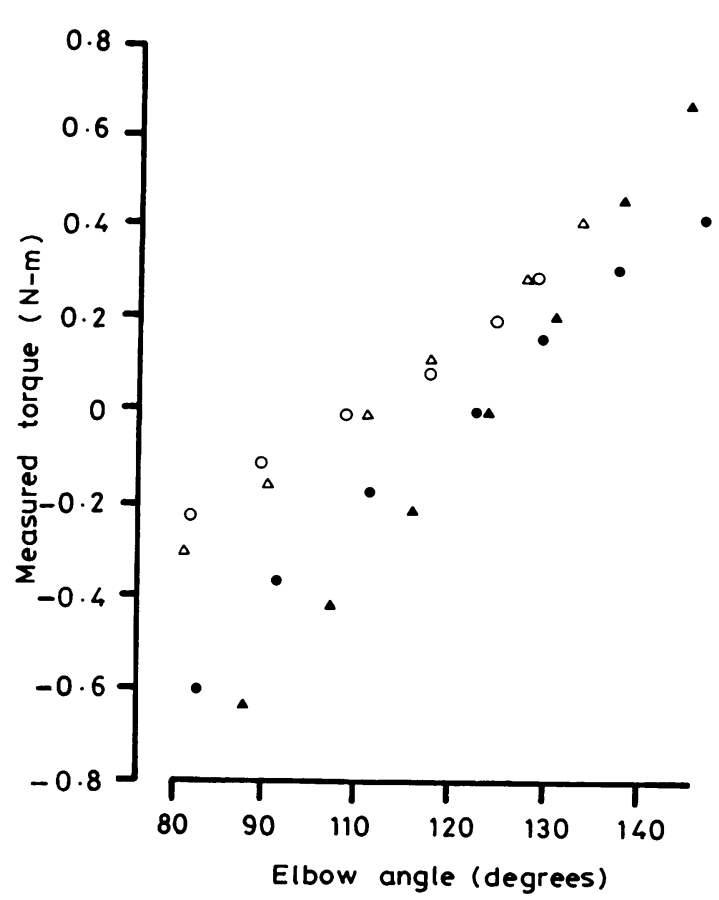

Fig 4 Reproducibility of measured torque versus elbow angle data was assessed in subjects 1 (open markers) and 3 (closed markers) by testing them on two different days. Note the essentially linear relation obtained from each test. The slope of the linear regression of angle on torque is the limb's compliance. The average correlation coefficient from these 4 regressions was 0.995 , which is equal to the mean for all subjects tested. 
TABLE: Data summary, 19 subjects

\begin{tabular}{|c|c|c|c|c|c|c|c|}
\hline Subject & Sex & Age (yr) & $\begin{array}{l}\text { Regular } \\
\text { Compliance } \\
(\% / \mathrm{Nm})\end{array}$ & $\begin{array}{l}\text { Frictionless } \\
\text { Compliance } \\
(\% / \mathrm{Nm})\end{array}$ & $\begin{array}{l}\text { Stiffness } \\
(\mathrm{Nm} / \mathrm{rad})\end{array}$ & $\begin{array}{l}\text { Upper Arm } \\
\text { Volume }\left(\mathrm{cm}^{3}\right)\end{array}$ & $\begin{array}{l}\text { Neutral Elbow } \\
\text { angle (degrees) }\end{array}$ \\
\hline $\begin{array}{l}1 \\
2 \\
3 \\
4 \\
5 \\
6 \\
7 \\
8 \\
9 \\
10 \\
11 \\
12 \\
13 \\
14 \\
15 \\
16 \\
17 \\
18 \\
19 \\
\text { Mean } \\
\pm \text { SD }\end{array}$ & $\begin{array}{l}\mathbf{M} \\
\mathbf{M} \\
\mathbf{M} \\
\mathbf{M} \\
\mathbf{F} \\
\mathbf{M} \\
\mathbf{M} \\
\mathbf{F} \\
\mathbf{F} \\
\mathbf{F} \\
\mathbf{M} \\
\mathbf{M} \\
\mathbf{F} \\
\mathbf{M} \\
\mathbf{M} \\
\mathbf{M} \\
\mathbf{F} \\
\mathbf{F} \\
\mathbf{F}\end{array}$ & $\begin{array}{l}35 \\
24 \\
29 \\
48 \\
28 \\
32 \\
36 \\
38 \\
62 \\
22 \\
20 \\
30 \\
22 \\
59 \\
78 \\
70 \\
22 \\
23 \\
51\end{array}$ & $\begin{array}{r}81 \\
51 \\
46 \\
55 \\
140 \\
52 \\
35 \\
76 \\
73 \\
171 \\
30 \\
52 \\
54 \\
32 \\
82 \\
54 \\
72 \\
138 \\
50 \\
71 \\
\pm 39\end{array}$ & $\begin{array}{c}74 \\
63 \\
52 \\
50 \\
121 \\
65 \\
39 \\
74 \\
73 \\
144 \\
36 \\
44 \\
67 \\
32 \\
86 \\
48 \\
107 \\
145 \\
46 \\
72 \\
\pm 34\end{array}$ & $\begin{array}{r}0.77 \\
0.91 \\
1.10 \\
1.15 \\
0.48 \\
0.88 \\
1.47 \\
0.77 \\
0.78 \\
0.40 \\
1.59 \\
1.30 \\
0.85 \\
1.79 \\
0.66 \\
1.19 \\
0.53 \\
0.40 \\
1.24 \\
0.96 \\
+0.40\end{array}$ & $\begin{array}{l}1950 \\
2090 \\
2470 \\
2390 \\
1360 \\
1620 \\
2380 \\
2040 \\
1570 \\
1380 \\
2740 \\
2380 \\
1410 \\
3130 \\
2360 \\
1880 \\
1200 \\
1450 \\
2340 \\
2010 \\
\pm 540\end{array}$ & $\begin{array}{r}101 \\
120 \\
114 \\
94 \\
108 \\
107 \\
114 \\
130 \\
119 \\
111 \\
100 \\
93 \\
87 \\
111 \\
106 \\
102 \\
111 \\
101 \\
108 \\
107 \\
\pm 10\end{array}$ \\
\hline
\end{tabular}

compliance and multiplying by $360 / 2 \pi$. At this point friction was either restored or cancelled and a second block of trials performed.

Upper arm volume was estimated by recording the circumference of the arm at three points equidistant between the shoulder and elbow, and by measuring the length of the arm from the acromion to the elbow. After averaging the circumferences we modelled the upper arm as a cylinder and calculated its volume.

\section{Results}

Nineteen subjects, 11 males and eight females, successfully completed the study (table). They ranged in age from 20 to 78 years with a median of 32 . Compliance curves from repeated trials on two subjects are shown in fig 4. Subject 1 (open markers) and subject 3 (closed markers) were each tested twice, each test on a separate day. Figure 4 demonstrates both the linearity of the relation between torque and elbow angle over a range of $\pm 30^{\circ}$ from the neutral angle, and the reproducibility of the test from day to day. The slopes of the lines fitted to this data, and similar data from other subjects, are shown in the columns labeled "regular compliance" and "frictionless compliance" in the table, the latter from the blocks of trials in which friction was electronically cancelled. Regular compliance ranged from 30 to $171^{\circ} / \mathrm{Nm}$ and frictionless compliance from 32 to $145^{\circ} / \mathrm{Nm}$; there was no consistent difference between the two measures and frictionless compliance will be used hereafter. The smallest correlation coefficient among all of the linear regressions used to determine compliance was $\mathbf{0} \cdot \mathbf{9 8 6}$. Compliance was significantly correlated $(r=-0.76, p<0.01)$ to upper arm volume (fig 5A), which reflects the mass of the muscles acting at the elbow. Neither compliance nor upper arm volume was correlated with age. Neutral position of the forearm (the resting elbow angle in absence of applied torque) ranged from $87^{\circ}$ to $130^{\circ}$ with a mean \pm SD of $107 \pm 10^{\circ}$.

The apparently hyperbolic relation between compliance (C) and upper arm volume (V) suggested the following equation relating the variables:

$$
\mathrm{C}\left(\mathrm{V}-\mathrm{V}_{0}\right)=\mathrm{K}
$$

where $\mathrm{V}_{0}$ is an asymptote corresponding to a volume within the arm consisting of non-elastic structures. Equation 1 can be linearised by expressing compliance as its inverse, stiffness (S) in $\mathrm{Nm} /$ radian $\left(1\right.$ radian $\left.=57.3^{\circ}\right)$ :

$$
\begin{gathered}
(1 / S)\left(V-V_{0}\right)=K \\
\text { or, } S=(1 / K) V-V_{0} / K
\end{gathered}
$$

The compliance data in the table were converted to their stiffness equivalents and a linear regression line fitted to the data. The result was:

$$
\mathrm{S}=-0.32+0.00064 \mathrm{~V}
$$

with a correlation coefficient of 0.85 and standard error of estimate (SEE) $=0.22$. From Equations 3 and 4 one can calculate $\mathrm{V}_{0}=500 \mathrm{~cm}^{3}$. Figure $5 \mathrm{~B}$ shows the fitted linear regression line and 95\% confidence limits for predicted stiffness at a given arm volume. ${ }^{11}$ 


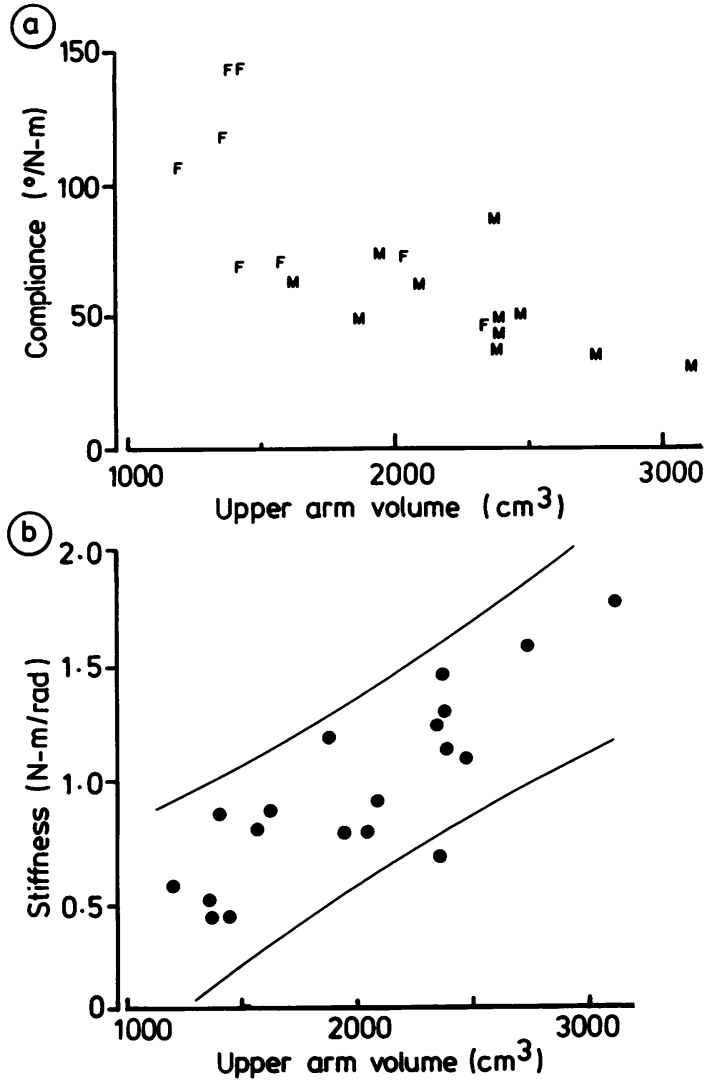

Fig 5 (a) Relation between compliance at the elbow and upper arm volume in the absence of muscle activity. Correlation was significant $(r=-0.76, p<0.01)$ with male subjects (M) having lower compliance values and larger arm volumes than female subjects $(F)$. (b) Inverting compliance to stiffness linearises the above relation. The regression line fitted to all 19 subjects is given in text Eqn. 4. The curved lines represent $95 \%$ confidence limits for normal data.

\section{Discussion}

Quantitatively, compliances obtained in the present study agree with those of Fel'dman ${ }^{1}$ (1 measurement, $45^{\circ} / \mathrm{Nm}$ ) and Hayes and Hatze ${ }^{2}$ (three measurements, 41,41 , and $57^{\circ} / \mathrm{Nm}$, converted to compliance). Compliance is found to have a large range of values in normal subjects, probably because it is a function not only of the properties of muscle, but also of the mass (or more precisely, cross-sectional area) of muscles acting at the elbow. Thus one expects compliance to be correlated with upper arm volume, as indeed it is.

Our use of upper arm volume to estimate muscle area obviously did not take into account the volume occupied by bone and adipose tissue. $V_{0}$, the non- elastic volume, was found to be $500 \mathrm{~cm}^{3}$, which is equivalent to a cylinder $4.4 \mathrm{~cm}$ in diameter within the upper arm (somewhat larger than the volume of the humerus). More accurate estimates of muscle mass could be obtained using a triceps skinfold technique ${ }^{12}$ to estimate adipose tissue content, or, ultimately, by using a computed tomography (CT) scan at the biceps level. CT scanning would have added substantially to the cost of the study and subjected the subjects to a small dose of radiation, without assurance of a significantly improved correlation.

The linearity of the angle versus torque relation found here is a consequence of our analysis over a limited angular range, approximately $\pm 30^{\circ}$ from the neutral position. The exponential length-force relation of muscle and the hard limits imposed by joint structures cause the angle-torque relation to become non-linear for large excursions, as can be seen in the data of Hayes and Hatze. ${ }^{2}$ By limiting our study to the middle of the range, we characterise compliance with a single number and minimise other complexities such as shortening reactions which are more common at full extension or flexion.

Shortening reactions or responses, that is EMG activity in a muscle being passively shortened, have been observed for a long time ${ }^{10}$ but not widely reported. They may act to prevent buckling of muscle fibres and consequent loss of sensitivity of muscle spindle primary endings as a muscle is passively shortened to its slack length. Norton and Sahrmann ${ }^{13}$ found biceps or brachioradialis shortening responses in a majority of normal subjects. In the present study, shortening responses in the triceps were observed in a number of subjects; most subjects could eliminate the response by concentrating on total relaxation of the limb. Occasional shortening responses were handled by repeating trials in which they occurred. Three subjects were removed from the study because of intractable shortening responses. To the extent that any shortening responses went undetected, they would tend to increase the apparent compliance at the elbow.

Neutral elbow angle has been reported to be $122 \pm 24^{\circ}$ by the National Aeronautics and Space Administration ${ }^{14}$ for persons under weightless conditions, while here on earth, Pertuzon and Lestienne ${ }^{15}$ found an equilibrium position of $75^{\circ}$ from full extension ( $105^{\circ}$ angle at elbow). Interestingly, this equilibrium point has also been found to be the point of minimum viscous stiffness. ${ }^{2}$

Thus, we have defined a simple method for quantifying resting "tone", or stiffness, at the elbow in normal subjects, as well as defining the neutral angle of the relaxed limb. Measurements of upper arm volume were used to reduce the variance of the data and allowed us to set up reasonably narrow limits for 
"normal" tone. We can compare these normal values for tone with those obtained from patients with rigidity ${ }^{8}$ or spasticity in order to determine to what extent this increased tone is a consequence of altered muscle properties as opposed to enhanced reflexes.

We appreciate the technical assistance of $\mathrm{Mr}$ Jonathan Holder and Ms. Judith Warner.

This work was supported in part by the Massachusetts General Hospital Fund for the Study of Parkinson's Disease and Related Conditions. Dr Watts is currently a Medical Staff Fellow in the Laboratory of Neurophysiology, NIMH, Bethesda, MD.

\section{References}

${ }^{1}$ Fel'dman AG. Functional tuning of the nervous system with control of movement or maintenance of a steady posture-II. Controllable parameters of the muscles. Biofizika 1966;11: No 3, 498-508.

${ }^{2}$ Hayes KC, Hatze H. Passive visco-elastic properties of the structures spanning the human elbow joint. Eur J Appl Physiol 1977;37:265-74.

${ }^{3}$ Bizzi E, Accornero N, Chapple W, Hogan N. Arm trajectory formation in monkeys. Exp Brain Res 1982;46:139-43.

${ }^{4}$ Lakie M, Walsh EG, Wright GW. Resonance at the wrist demonstrated by the use of a torque motor: an instrumental analysis of muscle tone in man. $J$ Physiol (Lond) 1984;353:265-85.

${ }^{5}$ Marsden CD, Obeso JA, Rothwell JC. The function of the antagonist muscle during fast limb movements in man. J Physiol (Lond) 1983;335:1-13.

${ }^{6}$ Dietz V, Quintern J, Berger W. Electrophysiological studies of gait in spasticity. Brain 1981;104:431-49.

${ }^{7}$ Wiegner AW. Thixotropic behavior at joints has its origin in short range stiffness of relaxed muscle. Biophys $J$ 1985;47:291a.

${ }^{8}$ Watts RL, Wiegner AW, Young RR. Elastic properties of muscles measured at the elbow in man: II. Patients with Parkinsonian rigidity. $J$ Neurol Neurosurg Psych 1986;49:1177-81.

${ }^{9}$ Gossman MR, Sahrmann SA, Rose SJ. Review of lengthassociated changes in muscle. Phys Ther 1982;62:12, 1799-1830.

${ }^{10}$ Westphal C. Ueber eine Art paradoxer Muskelcontraction. Arch Psychiat NervKrankh 1880;10:243-8.

${ }^{11}$ Sachs L. Applied Statistics. Second edition, New York: Springer-Verlag, 1984:444.

${ }^{12}$ Gerney JM, Jelliffe DB. Arm anthropometry in nutritional assessment: nomogram for rapid calculation of muscle circumference and cross-sectional muscle and fat areas. Am J Clin Nutr 1973;26:912-5.

${ }^{13}$ Norton BJ, Sahrmann SA. Shortening responses in normal, spastic and rigid muscle. Soc Neurosci Abstr 1983;9: No 1, 632.

${ }^{14}$ NASA Anthropometric Source Book, Vol I Anthropometry for Designers. NASA Ref Publication 1978;1024:I20-6.

${ }^{15}$ Pertuzon E, Lestienne F. Determination dynamique de la position d'equilibre d'une articulation. Int $Z$ Angew Physiol 1973;31:315-25. 